\title{
Early environmental enrichment protects captive-born striped mice against the later development of stereotypic behaviour
}

\author{
Megan Anne Jones ${ }^{\mathrm{a}, *}$, Georgia Mason ${ }^{\mathrm{a}, \mathrm{b}}$, Neville Pillay ${ }^{\mathrm{a}}$ \\ a School of Animal, Plant, and Environmental Sciences, University of the Witwatersrand, Private Bag 3, WITS, 2050, South Africa \\ b Department of Animal and Poultry Sciences, University of Guelph, 50 Stone Road East, Guelph, Ontario, Canada N1G 2W1
}

\section{A R T I C L E I N F O}

\section{Article history:}

Accepted 26 August 2011

Available online 1 October 2011

\section{Keywords:}

Birth origin

Enrichment

Frustration

Neuroprotection

Rhabdomys

Stereotypic behaviour

Welfare

\begin{abstract}
A B S T R A C T
Understanding how birth origin (whether born in the wild or captivity) influences behavioural development is important for fundamental and applied ethology, especially when captive-bred (CB) individuals from wild species are used in research or conservation. CB animals are typically much more prone to stereotypic behaviour (SB) than are wild caught (WC) conspecifics, an effect which in striped mice is accompanied by increased tendencies to form behavioural routines. However, WC mice, if stereotypic, are far more severely affected than $C B$ mice and are also more physiologically stressed and inactive than $\mathrm{CB}$ mice, regardless of their stereotypy status. Capturing subjects from the wild to further study these latter effects raises serious practical, ethical and potentially conservation concerns. Here, we therefore tested whether rearing $C B$ striped mice in enriched conditions and then placing them in standard cages could provide a more suitable model for investigating how wild-caught (WC) conspecifics respond to the reduced environmental complexity they experience after capture. Compared with striped mice which were always standard-housed ( $n=36)$, enriched striped mice $(n=24)$ were four times less likely to develop SB and, similarly to the benefits of being wild born, early environmental enrichment (30-170 days) successfully protected CB striped mice from the emergence of SB after transfer to standard caging (171-240 days). However, unlike WC mice, previously enriched CB striped mice which then became stereotypic did not develop markedly more severe SBs; they showed diverse forms, unlike stereotypic WC mice which exclusively circuit ran; and they were not more inactive once SB levels were controlled for. These findings thus show that early environmental complexity can have lasting suppressive effects on SB, suggesting that early enrichment protocols could indeed provide a practicable, potentially more ethical model for investigating the causal mechanisms underpinning birth origin effects on $\mathrm{SB}$. Furthermore, they add to a corpus of data for this species showing that more naturalistic early experiences, both social and physical, lastingly protect against SB development. These results also highlight how unpredictable and poorly understood early enrichment effects are (since in some species early enrichment exacerbates later SB); and moreover reveal that early environmental complexity is not the sole factor shaping behavioural phenotypes in WC animals, with differential social experience and human contact being other likely causes of developmental divergence.
\end{abstract}

(C) 2011 Elsevier B.V. All rights reserved.

\footnotetext{
* Corresponding author. Tel.: +27 11717 6400; fax: +27 114031429.

E-mail address: megan.jones@icon.co.za (M.A. Jones).
}

\section{Introduction}

Stereotypic behaviours (SBs) are rife in animals housed in restricted and impoverished environments (Mason and Latham, 2004), but typically less prevalent and 
time-consuming in more complex, enriched conditions (Mason et al., 2007). In general, situations inducing or exacerbating SBs also decrease welfare (Mason and Latham, 2004). The performance of SBs suggests the thwarting of highly motivated behaviours (Latham and Mason, 2010) and/or neurodevelopmental disruption to pathways of the forebrain and basal ganglia which subserve the control of normal, flexible behaviour (Garner, 2006; Lewis et al., 2006; Latham and Mason, 2010). In captive wild animals (vs. captive domestic animals), SBs are especially common in those individuals born and reared in captivity, most likely because captive-born (CB) animals experience earlier and/or more prolonged deprivation than do conspecifics captured from the wild (i.e. wild-caught [WC] individuals; Mason, 2006). This effect of birth origin on SB development is evident in the behaviour of captive striped mice, Rhabdomys (Jones et al., 2011): striped mice which spend a prolonged period of time in the wild before capture are substantially less likely to stereotype than CB individuals. Their low SB is linked to increased behavioural flexibility under test compared to that of $\mathrm{CB}$ animals, indicating that nonstereotypic WC mice have relatively normal behavioural control. However, as a group, regardless of whether or not they are stereotypic, WC mice are more fearful than CB individuals, have higher corticosteroid outputs, and are concerningly far more inactive (Jones et al., 2011).

In contrast to WC animals, in which previous experiences in complex, natural environments typically protect against later SB development, the barren housing of formerly environmentally enriched $\mathrm{CB}$ animals has inconsistent long-term influences on SB performance. In some environmental enrichment (EE) studies, previously enriched animals (e.g. bank voles, Clethrionomys glareolus; Ödberg, 1987; deer mice, Peromyscus maniculatus; Lewis et al., 2006) remain, like WC individuals, less stereotypic after being transferred to barren cages than standardhoused controls-an effect which correlates, in deer mice, with altered dendritic morphology in the motor cortex and striatum (Turner et al., 2003), reduced striatal enkephalin, a marker for indirect pathway activity (Presti and Lewis, 2005), and high cytochrome oxidase activity in the substantia nigra in the motor cortex and basal ganglia, an index of activity-dependent plasticity and neuronal metabolic activity (Turner et al., 2002).

In contrast, other EE studies have found the opposite effect: for instance, SB incidence and severity is higher in laboratory mice (Mus musculus) from which EE is removed than in mice which have always been deprived, and its exacerbation in previously enriched mice is statistically associated with evidence of heightened frustration (Latham and Mason, 2010). The unpredictable response of $\mathrm{CB}$ enriched-reared animals to an environmental downshift (i.e. transfer from rich, complex conditions to comparatively impoverished housing) likely reflects the relative influences of motivational and neurodevelopmental factors. When an environmental downshift is associated with continuing low levels/incidences of SB, the motivational sequelae of the downshift (i.e. negative contrast/frustrative non-reward) are arguably weaker determinants of behaviour than is intact forebrain function, which protects against SB development ('Protection
Hypothesis': Latham and Mason, 2010). In contrast, when an environmental downshift exacerbates SB performance, it presumably does so by means of frustration, which motivates SB performance in individuals for which the previous environmental complexity was insufficient to normalize forebrain development ('Frustration Hypothesis': Latham and Mason, 2010).

Understanding how and why previous experience in complex environments influences SB development is of central concern to fundamental and applied ethologists. For example, in captive wild-derived animals, better understanding of the influences of early experience on adult phenotype is necessary for multiple reasons: practically, birth origin differences in SB incidence indicate an undesirable divergence of behavioural phenotype between WC and $\mathrm{CB}$ individuals. This divergence raises concerns about the validity of ethological studies using $C B$ animals to model behavioural processes in WC animals as well as the current management of wild animals in captive-breeding and release programmes. Understanding birth origin effects is the first step towards ameliorating these concerns. However, capturing animals from the wild to investigate birth origin effects, raises other practical, ethical and, for many species, conservation concerns. Retrospective analysis of historical data sets (e.g. Jones et al., 2011), which avoids these issues, provides some insights into the correlates of birth origin effects, but the results from such analyses do not necessarily provide causal insights and are limited by the variables recorded. Furthermore, catching new animals from the wild precludes the investigation of some research questions principally because the experiences of WC animals before capture are unknown and cannot easily be manipulated, making it difficult or impossible to tease apart the relative contributions of early social environment, physical environment, and/or prior lack of exposure to humans on their subsequent responses to captivity. Rearing CB animals in enriched and complex captive environments and then placing them in standard barren cages might provide an alternative opportunity to assess how WC conspecifics respond to reduced environmental complexity, mimicking the transfer of WC animals from complex natural environments to barren captive environments. Such experiments could also allow for the experimental manipulation of candidate variables and thus, ultimately, elucidation of the mechanisms underpinning the effects of birth origin and early experience on SB.

In this study, we therefore investigated the outcomes of an EE paradigm on the emergence of SB in F1 CB striped mice. This research was needed because, as reviewed above, early enrichment cannot always be assumed a priori to have effects similar to those of being WC. We compared the incidence of SB in CB striped mice housed from shortly after weaning (30 days) until late adulthood in standard laboratory cages with the incidence of SB in CB striped mice housed in larger and more complex cages (Phase 1 ). Thereafter, we transferred the previously enriched striped mice to standard caging and, 10 weeks later, again compared the incidence of SB between the two treatment groups (Phase 2 ). Because little is known about how cage characteristics influence the form of SB (Würbel, 2006), and because WC mice that stereotype exclusively circuit run, we also 


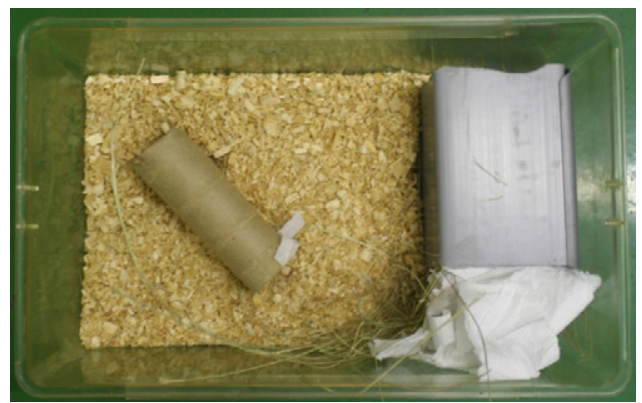

Fig. 1. The standard Labotec ${ }^{\mathrm{TM}}$ cage in which breeding pairs and standardhoused striped mice were kept. The cage contained woodshavings, a PVC tubing nest box provisioned with hay and paper towelling, and one cardboard roll.

recorded preferred SB form both before and after transfer to standard caging. Finally, given that non-stereotypic WC striped mice are very inactive, and that stereotypic WC striped mice have markedly time-consuming SBs (Jones et al., 2011; responses suggestive of fear and frustration respectively), during Phase 2 we additionally recorded the proportion of time that striped mice spent engaged in nonstereotypic activity in the home cage, or performing SB in the nest.

\section{Methods and materials}

\subsection{Subjects}

CB striped mice (males: $n=32$; females: $n=28$ ) used in this study were a randomly selected group of F1 offspring from $10 \mathrm{WC}$ breeding pairs. The WC founder stock, which originated from a Highveld grassland locality (Gauteng, South Africa; $27^{\circ} 55^{\prime} \mathrm{S}, 26^{\circ} 4^{\prime} \mathrm{E}$ ), were trapped for use in other behavioural studies (see Jones et al., 2011).

\subsection{Housing and husbandry}

Striped mice were housed in either the Milner Park Animal Unit at the University of the Witwatersrand, under partially controlled environmental conditions (14L:10D, lights on at $05: 00 \mathrm{~h} ; 20-24^{\circ} \mathrm{C} ; 30-60 \%$ relative humidity) or, because of the extra space required for the enriched housing, in a room in the adjacent Biology Building (14L:10D, lights on at 05:00 h; ambient temperature and humidity). Breeding pairs and standard-housed experimental striped mice (all located in the Milner Park Animal Unit) were kept in Labotec ${ }^{\mathrm{TM}}$ cages $(300 \mathrm{~mm} \times 200 \mathrm{~mm} \times 150 \mathrm{~mm})$ containing $\sim 2 \mathrm{~cm}$ woodshavings as bedding; a PVC tubing nest box $(10 \mathrm{~cm} \times 10 \mathrm{~cm} \times 15 \mathrm{~cm}$, open at both ends $)$ provisioned with a grass mix (Eragrostis grass, oat hay, and/or lucerne) and paper towelling as nesting material; and one cardboard roll (Fig. 1). Enriched-housed striped mice (about half of which were housed in the Milner Park Animal Unit and half in the Biology Building) were kept in large tanks ( $460 \mathrm{~mm} \times 300 \mathrm{~mm} \times 320 \mathrm{~mm}$ high) with three metal sides, a clear perspex front, and a wire mesh lid; the tanks were connected by a $20 \mathrm{~cm}$ long white PVC tube $(5 \mathrm{~cm}$ diameter) to a small cage $(20 \mathrm{~mm} \times 150 \mathrm{~mm} \times 150 \mathrm{~mm})$ in which food and water were provided (Fig. 2). The large

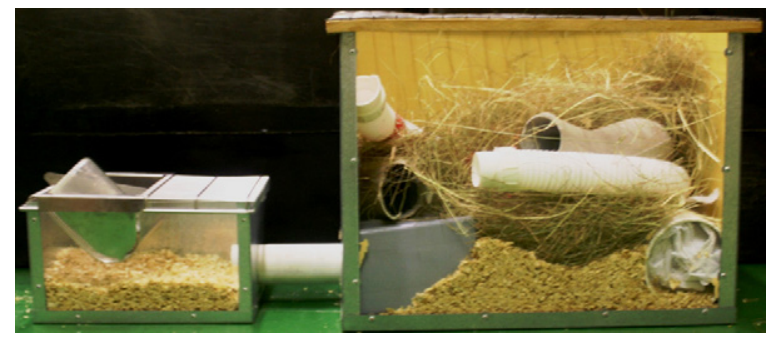

Fig. 2. The enriched tank connected by a PVC tube to a small Labotec ${ }^{\mathrm{TM}}$ cage, in which enriched-housed striped mice were kept. The tank contained $\sim 5 \mathrm{~cm}$ woodshavings as bedding, another $\sim 15 \mathrm{~cm}$ of a hay mixture, a PVC tubing nest box provisioned with hay and paper towelling, between 3 and 5 cardboard rolls, and a $1 \mathrm{~m}$ long flexible plastic pipe. The small cage contained $\sim 2 \mathrm{~cm}$ of woodshavings.

tank contained a deep layer of woodshavings as bedding $(\sim 5 \mathrm{~cm})$ covered by an additional $\sim 15 \mathrm{~cm}$ layer of a grass mix. The tank was also provisioned with a PVC tubing nest box containing paper towelling as bedding; $1 \mathrm{~m}$ long, $5 \mathrm{~cm}$ diameter, flexible plastic pipe; and between 3 and 5 cardboard rolls. Both standard-housed and enriched-housed striped mice had access ad libitum to Epol mouse cubes and water. Each day, a small amount $( \pm 3 \mathrm{~g})$ of seed mix (sunflower, millet, canary) was sprinkled in the cages/tanks to encourage foraging, and fresh fruit and/or vegetables $( \pm 10 \mathrm{~g})$ were provided.

\subsection{Procedure}

Captive-born F1 striped mice were separated from their dams at 22 days of age and housed until 30 days old in their sibling groups in large tanks (the same as for the enriched-housing, but not connected to a small cage; Fig. 3). Thereafter, striped mice were pseudo-randomly assigned to individual housing in one of two treatment groups (balancing animals across treatment groups for litter and for sex): ( 1 ) enriched-housed/standard-housed ( $E / S ; n=24)$ or (2) standard-housed/standard-housed ( $S / S ; n=36)$. Striped mice in the $E / S$ treatment were first housed in enriched tanks for 140 days (between 30 and 170 days of age; Phase 1) after which they were transferred to standard cages for another 70 days (171-240 days; Phase 2). Individuals in the $\mathrm{S} / \mathrm{S}$ treatment were housed in standard cages for the entire 210-day experimental period (i.e. during both Phases 1 and 2).

\subsubsection{SB incidence and form}

We defined SB as a repetitive behaviour comprising at least three successive repetitions (Mason, 1993; Vickery and Mason, 2004; see Table 1 for definitions of the various forms of SBs observed). During Phase 1 (30-170 days), individual striped mice were assessed every day for 140 days for SB in their home cages using ad libitum sampling (when a striped mouse was seen performing $S B$, the presence and the form of the SB displayed was recorded on its cage tag by MJ). These direct observations were corroborated using video-recording of home cage behaviour (no observer present), for $20 \mathrm{~min} /$ day, on three non-consecutive days, between days 161 and 170 . Thereafter, during Phase 2 (171-240 days), the home cage behaviour of striped mice 


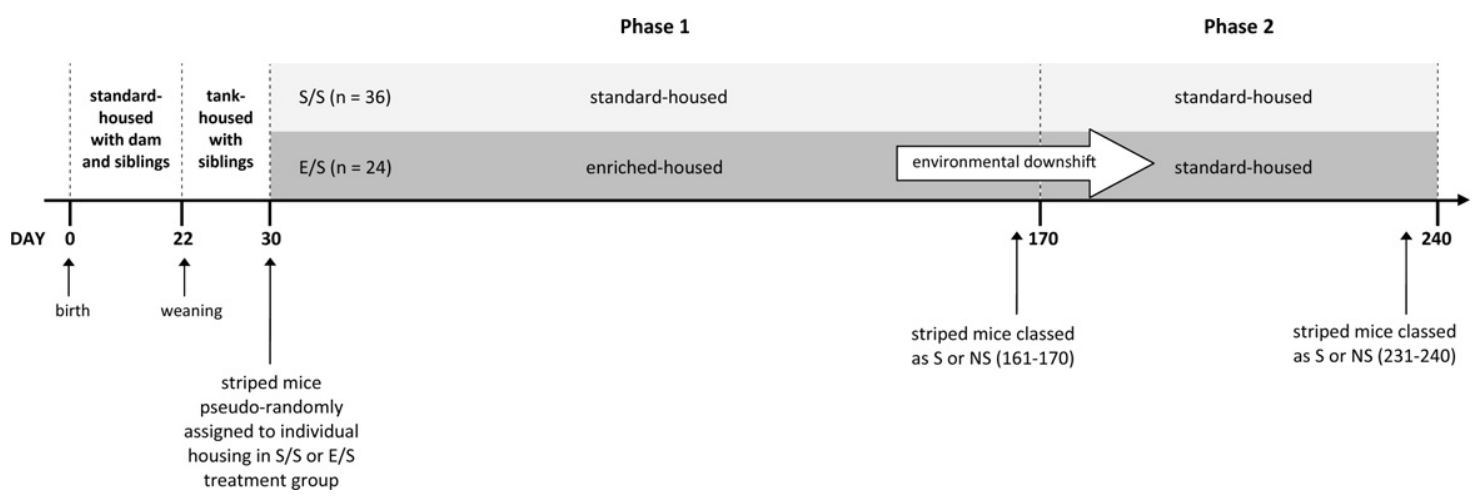

Fig. 3. Timeline indicating age (days) at which striped mice were weaned, assigned to either the S/S or E/S treatment group, and kept in Phase 1 or 2 housing.

was observed for 5 days/week, for 10 weeks, for $15 \mathrm{~min} /$ day, using direct behavioural observations (by MJ) and, on three randomly chosen days between days 231 and 240, their behaviour was video-recorded for $20 \mathrm{~min} /$ day. Striped mice which never displayed SB during Phases 1 or 2 of the experiment were classified as non-stereotypic (NS) for that phase, whereas those animals displaying SB during that time period were classified as stereotypic (S; see Jones et al., 2010a,b, 2011; for a justification of this dichotomous classification method).

An individual's preferred form of SB during Phase 1 was roughly evaluated (c.f. Phase 2 ) based on the written recordings made on each striped mouse's cage tag: if only a single form of SB had been documented, that SB form was designated as the preferred form. If, however, more than one form of SB had been displayed (irrespective of how frequently each form was performed), we designated the preferred form of SB as 'mixed'. In Phase 2, using a more rigorous method, we determined the preferred form of SB for stereotypic mice by dividing the total number of times a particular SB was shown by the total number of days on which any SB was observed (more than one form of SB could be recorded within one observation session). If a particular SB was observed in more than $70 \%$ of the observation sessions (provided the sum of the second and third most common forms did not exceed $70 \%$ of the observation sessions), that form of SB was regarded as the preferred form. If no SB form was performed in more than $70 \%$ of observation sessions, or if the sum of the second and third most common forms exceeded $70 \%$, then the preferred form of SB was designated as 'mixed'.

\subsubsection{Phase 2 home cage behaviour}

During Phase 2 only, we calculated the proportion of observation days on which striped mice either (1) did not leave their nest box; (2) were active outside the nest box (locomotion, grooming, drinking, eating, but excluding SB); or (3) performed SB (i.e. mutually exclusive categories).

\subsection{Data analysis}

We ran analyses using either SPSS (Version 18) or Statistica (Version 8.0).

\subsubsection{SB incidence}

To assess the effects of treatment on the incidence of SB between the two treatment groups, we used a Generalized Linear Model (GLZ) with a repeated measures design, followed by pair-wise planned contrasts (SPSS). In the model, we included treatment and sex as categorical predictors; phase (Phase 1 or 2 ) as the repeated measure; and the stereotypic status (S or NS) of each striped mouse as the dependent variable. We nested housing location (Milner Park Animal Unit/Biology Building) within treatment to account for the potential effects of differential housing location during Phase 1.

\subsubsection{SB form}

For Phases 1 and 2 separately (since categorization of preferred form was not directly comparable), we assessed the preferred form of SB between treatment groups using a GLZ with treatment group as the categorical predictor, and the frequency of each form of SB as the response variable (Statistica).

\subsubsection{Phase 2 home cage behaviour}

First, to examine between-group differences in home cage behaviour, we compared the proportion of observation sessions (arcsine square root transformed; Zar, 1996) in which striped mice did not leave the nest box; were

Table 1

Descriptions of the four main forms of SB observed.

\begin{tabular}{ll}
\hline Stereotypic behaviour & Description \\
\hline Circuit running & Running in the cage along a fixed route. \\
Prelooping & $\begin{array}{l}\text { Climbing upside down on the cage lid with forelimbs and then hindlimbs, moving backwards along lid, } \\
\text { followed by dropping down through releasing the hindlimbs first. }\end{array}$ \\
Somersaulting & Backward flipping, with or without touching the cage. \\
Windscreen wiping & Hindlimbs remain stationary on the cage floor, or move far less than do forelimbs. Forelimbs oscillate \\
& to-and-fro in a large arc against the front/side of the cage, or the food hopper.
\end{tabular}




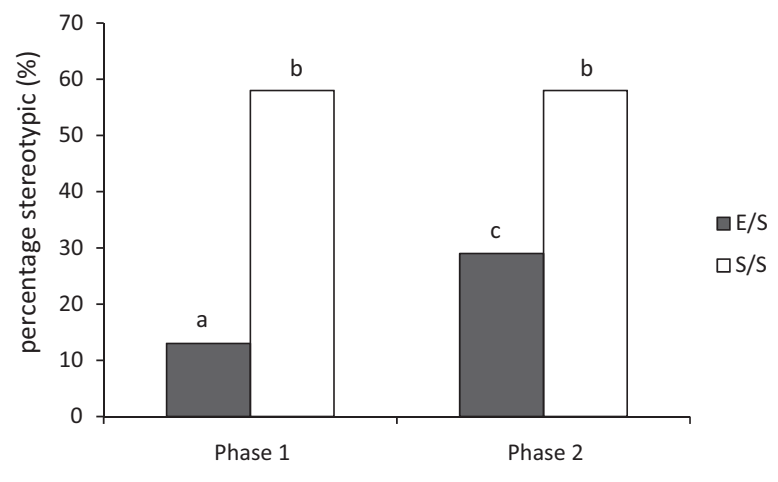

Fig. 4. Prevalence of stereotypic behaviour in striped mice reared in enriched and standard housing (Phase 1), and after transfer of enrichedhoused animals to standard housing (Phase 2). Alphabet letters indicate homogenous groups.

active outside the nest; and in which individuals performed SB, using separate General Linear Models (GLM), including sex, treatment group, and previous housing location, which was nested within treatment group, as the categorical predictors (Statistica). Second, to assess whether previous housing type (enriched or standard) or current SB status was a better predictor of the proportion of observation sessions in the nest box and of non-stereotypic activity outside the nest, we re-ran the above GLMs, but included SB status as a categorical predictor. Third, we compared the severity of the SBs of stereotypic striped in the E/S and S/S groups using a GLM with sex, treatment group, and previous housing location nested within treatment group, as the categorical predictors, and the proportion of observation days on which stereotypic striped mice displayed SBs (arcsine square root transformed) as the dependent variable. Statistical findings were considered significant if $P \leq 0.05$.

\subsection{Ethical note}

Individual housing is ethically acceptable for this species, unlike many other rodents, because grasslandderived animals are naturally solitary. Striped mice used in this study were ultimately either euthanized with an overdose of an inhalant anaesthetic (Isoflurane or Halothane) or returned to breeding stock. Approval for this study was provided by the Animal Ethics Screening Committee of the University of the Witwatersrand (2006/94/03).

\section{Results}

\subsection{SB incidence}

Treatment (Wald $\chi^{2}=9.261 ; P=0.002$ ), phase (Wald $\chi^{2}=4.400 ; \quad P=0.036$ ), and their interaction (Wald $\chi^{2}=4.400 ; P=0.036$ ) were all significant predictors of the incidence of SB. At the end of Phase 1, whilst still in initially different housing conditions, striped mice from the E/S group (thus still in enriched cages) were significantly less likely to perform SB (3 out of $24 ; 13 \%$ stereotypic) than individuals housed in standard cages (21 out of 36 ; 58\% stereotypic) (pairwise comparison; $P<0.001$; Fig. 4). During Phase 2, when all striped mice were standard-housed, individuals from both treatments that had displayed SB in Phase 1 continued to stereotype. In the $S / S$ treatment group, no further animals developed SBs (thus $42 \%$ remained non-stereotypic) but, in the $\mathrm{E} / \mathrm{S}$ group, an additional four mice became stereotypic (bringing the total to 7 out of $24 ; 29 \%$ ). This increase in the incidence of SB from Phase 1 to 2 in the E/S group was statistically significant $(P=0.032)$ but, nonetheless, the overall incidence in the E/S group at the end of Phase 2 was still significantly lower than in the $\mathrm{S} / \mathrm{S}$ group $(P=0.017)$. The effects of sex (Wald $\chi^{2}=0.119 ; P=0.730$ ) and housing location (Wald $\chi^{2}=0.011 ; P=0.916$ ) on the incidence of SB were not significant.

\section{2. $S B$ form}

During Phase 1, the three stereotypic enriched-housed striped mice all showed circuit running (and no other forms of SB), whereas the 21 stereotypic S/S striped mice showed considerable intra- and inter-individual variation in their SB form: $62 \%$ circuit running ( $n=13$ ), $10 \%$ prelooping $(n=2), 5 \%$ somersaulting $(n=1), 5 \%$ windscreen wiping $(n=1)$, and $19 \%$ mixed $(n=4)$ (between-group differences in form; Wald $\chi_{4}^{2}=10.342 ; P=0.035$ ). During Phase $2, \mathrm{~S} / \mathrm{S}$ striped mice who were stereotypic during Phase 1 continued thus, and did not deviate from their established preferred forms of SB. In contrast, in the E/S group, two of the three previously identified circuit runners continued to display a preference for circuit running as did two of the four 'new' stereotypers (bringing the group total for circuit running to $57 \%$; $n=4$ ); one of the previous circuit runners switched to somersaulting (14\%; $n=1)$; and there was one new case of prelooping and one of mixed SB (14\%; $n=1$ respectively). Accordingly, during Phase 2 , and in contrast to Phase 1 , there were no differences between treatment groups in the preferred form of SB (Wald $\chi_{4}^{2}=$ $0.775 ; P=0.942$ ).

\subsection{Phase 2 home cage behaviour}

At a group level, E/S striped mice spent more time in the nest $\left(F_{1,56}=5.711 ; P=0.020\right)$ and performed less SB $\left(F_{1,56}=6.165 ; P=0.016\right)$ than did individuals in the $S / S$ treatment group (Fig. 5). E/S and S/S striped mice, however, showed comparable levels of non-stereotypic activity $\left(F_{1,56}=0.111 ; P=0.740\right)$. When we examined the concurrent effects of treatment group and stereotypy status on home cage behaviour, the main effects of treatment group disappeared (all $P>0.1$ ), and stereotypic status was the sole predictor of behavioural outcome (Fig. 6): stereotypic striped mice, irrespective of treatment group, spent less time in the nest $\left(F_{1,55}=196.087 ; P<0.001\right)$, and showed higher levels of non-stereotypic activity $\left(F_{1,55}=9.114\right.$; $P=0.004)$. There were no differences between treatment groups in the SB severity of stereotypic striped mice $\left(F_{1,24}=1.481 ; P=0.236\right)$ : thus, in both groups, stereotypic individuals performed similar levels of SB. In none of the above analyses was the response variable predicted by sex or Phase 1 housing location (all $P>0.1$ ). 


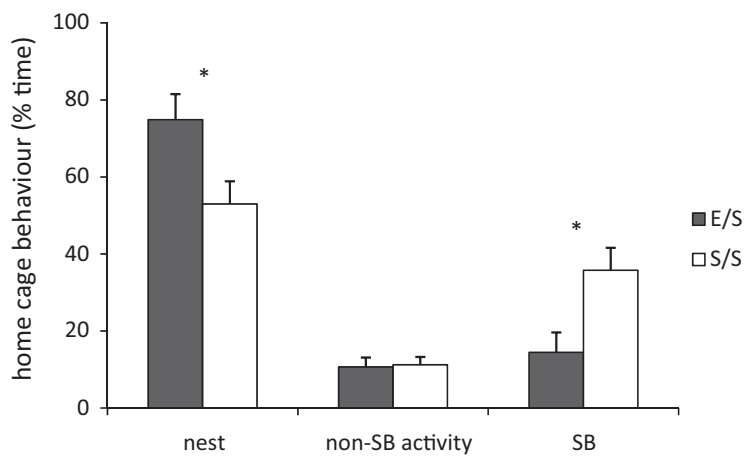

Fig. 5. Percentage of total observation sessions during Phase 2 in which striped mice from the E/S and S/S treatment groups were in the nest, engaged in non-stereotypic activity, or performing stereotypic behaviour. $* P<0.001$.

\section{Discussion}

In this study, we aimed to investigate the outcomes of an EE paradigm on the emergence of SB in CB striped mice, and thus whether an enrichment protocol could provide a practical, ethical means for investigating the mechanisms underpinning the protective effects of birth origin on SB development in WC animals. When striped mice were housed in enriched tanks, individuals were over four times less likely to develop SB than standardhoused controls (Phase 1). Thus the enrichment proved highly effective at reducing the prevalence of SB. Moreover, after these previously enriched striped mice were subsequently kept in standard caging for just over 3 months, only about one-quarter of formerly non-stereotypic individuals developed SB; in consequence, the overall incidence of SB in the E/S treatment group at the end of Phase 2 remained significantly lower than in the S/S comparison group. These results are consistent with our first prediction that early enrichment confers striped mice with long-lasting protection against the emergence of SB in later life, and thus indicate that a CB EE-based model of birth origin effects does merit further investigation. They also show that the early physical environment alone can have lasting effects, even when social experiences and human

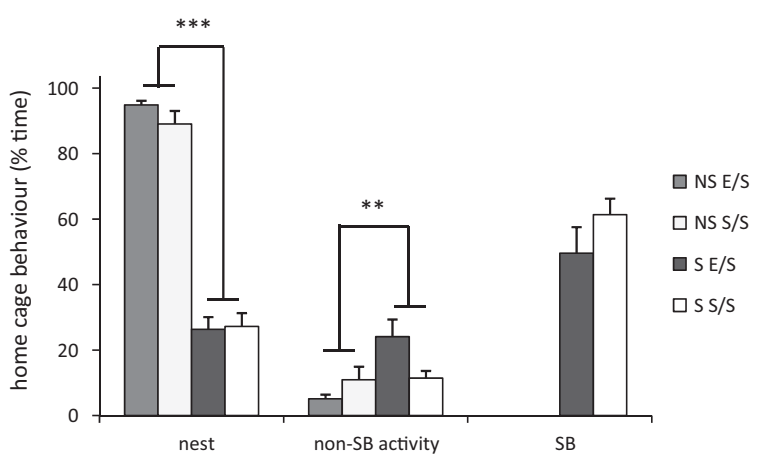

Fig. 6. Percentage of total observation sessions during Phase 2 in which striped mice were in the nest, engaged in non-stereotypic activity, or performing stereotypic behaviour in non-stereotypic (NS) and stereotypic (S) striped mice from the $\mathrm{E} / \mathrm{S}$ and $\mathrm{S} / \mathrm{S}$ treatment groups. ${ }^{* *} P<0.01{ }^{* * *} P<0.001$. contact are standardized, unlike the case in the WC vs. CB comparisons (Jones et al., 2011), so implicating early exposure to complex environments as one of the major causal factors underpinning the onset (or lack of onset) of SB.

However, early enrichment and its removal did not completely model the effects of being WC on SBs. For one, although enriched tanks greatly reduced the prevalence of SB whilst animals were living in such conditions, they did not abolish it entirely-a presumed difference in behaviour from life in the wild: SBs have not been observed in freeliving striped mice, and it is currently assumed SBs do not occur in the wild. Second, although early EE moderately successfully protected against the emergence of later SB in non-enriched cages, the prevalence of SB in such animals (7/24, 29\% stereotypic) was still significantly higher than the prevalence of SB in a random sample of WC adults ( $1 / 26 ; 4 \%$ stereotypic; Jones et al., 2011). Third, once moved to standard cages, the previously enriched striped mice and S/S individuals showed comparable interindividual variation in their predominant SB form; this contrasts with our previous work showing that WC striped mice developed only one form: circuit-running (Jones et al., 2011). The fourth and final difference is that those previously enriched striped mice that developed SB did not develop more time-consuming (severe) forms than did S/S individuals. Furthermore, early enrichment also did not completely model the effects of being WC on other aspects of behaviour. In particular, E/S animals were not more inactive than S/S striped mice, once levels of SB were statistically controlled for.

We hypothesise that the reduced incidence of SB in the E/S striped mice in the present study stems from environmentally mediated increases in behavioural flexibility, and thus that E/S striped mice, like their WC counterparts, will be less perseverative under test as a result of presumed intact forebrain function. Direct test of this hypothesis in future studies would further validate the use of an enrichment paradigm to model birth origin effects. We also suspect that these animals' greater SB prevalences than those of truly WC conspecifics reflect, at least in part, the limited physical complexity that is offered by enriched tanks compared to life in the wild. Tests of this hypothesis could include comparisons of CB mice raised in tanks vs. those reared even more naturalistically, for example in large outdoor enclosures. For the other main difference from WC mice-the lack of increased inactivity once SB was statistically controlled for-we hypothesise that this reflects that $\mathrm{E} / \mathrm{S}$ individuals are not motivated by fear to hide in the nest boxes, unlike WC animals, where inactivity correlates with raised levels of faecal corticosterone metabolites and more fearful behaviour in the light-dark box (proportion of time in the dark compartment; latency to emerge from the dark compartment; Jones et al., 2011). If this hypothesis is correct, we would expect E/S mice to be no more fearful in, for example, light-dark emergence tests than S/S mice, and for E/S mice to be likewise less fearful under test than WC mice. Since the high fearfulness of WC mice is likely to arise from a lack of habituation or even socialisation to humans, this hypothesis would also predict that raising $\mathrm{CB}$ mice in some automated system precluding 
human contact would yield adult subjects with the same fearful and inactive phenotypes as WC conspecifics.

As emphasised in the Introduction, removing environmental enrichments from animals has inconsistent effects on SB, with enrichment sometimes protecting them from later SB development, as seen here, whilst at other times dramatically increasing the incidence and severity of SB compared with animals that always have been barrenhoused. Currently there is insufficient knowledge to predict what effect will be seen with any given species or type of early enrichment: an intellectually unsatisfying situation. For future studies, we now propose two mediating variables as particularly likely to explain these discrepant findings:

(1) Differences in developmental stage when enrichment is both introduced and removed, in conjunction with the duration of exposure to the enrichment. The animal's developmental stage when enrichment is introduced/removed might influence whether animals (a) are motivated to interact with, and thus derive benefit therefrom (c.f. Tilly et al., 2010), and/or (b) whether environmentally induced changes to CNS structure and function are possible, since the benefits of enrichment on SB may be limited to, or be more effective, during 'sensitive periods' which typically occur earlier rather than later in ontogeny (Cooper et al., 1996; Hadley et al., 2006);

(2) The nature and degree of enrichment provided, which to date has varied from specific or modest additions to standard cages, through to ambitious "everything but the kitchen-sink" additions of multiple enrichments or even attempts to approximate natural conditions. In our study and the studies of Lewis and colleagues (e.g. Lewis et al., 2006)-where enrichment had an enduring effect on SB performance-enriched animals were provided with substantially larger cages than standardhoused controls (but c.f. Ödberg, 1987). These cages were also structurally complex, and contained a variety of natural and manufactured objects which could be explored, manipulated, and/or used for hiding. In comparison, in two of the studies (Vinke, 2004; Latham and Mason, 2010) where enrichment removal exacerbated the performance of SB, the complexity of enrichment was considerably less. As suggested by Latham and Mason (2010), enrichments might vary in their ability to normalize forebrain function and in their motivational salience, and thence the ability of enrichment to induce negative contrast and frustration at removal thereof.

The data reported here do support what is emerging as a prima facie general rule for striped mice, viz. the more relatively naturalistic their early rearing environment, the lower the incidence of SB in later life. This has been shown by manipulating the quality and quantity of parental care, as well as the complexity and duration of exposure to physically enriched environments (Table 2 ). In consequence, it is important for future studies to investigate the welfare correlates of low SB in all these subjects, including the $\mathrm{E} / \mathrm{S}$ animals of this experiment. Although 'low SB, good welfare'
Table 2

Incidence of SB of striped mice reared under less and more naturalistic conditions (less naturalistic; more naturalistic). Levels of SB are consistently and significantly lower in the more naturalistic treatment conditions.

\begin{tabular}{|c|c|c|}
\hline & \multicolumn{2}{|l|}{ Incidence of SB } \\
\hline & Less naturalistic & More naturalistic \\
\hline $\begin{array}{l}\text { Weaning age ( } 12 \text { days } \\
\text { vs. } 16 \text { or } 20 \text { days } \\
\text { old })^{\mathrm{a}}\end{array}$ & $\sim 68 \%(n=20)$ & $\sim 34 \%(n=20)$ \\
\hline $\begin{array}{l}\text { Parental care (only } \\
\text { mother vs. both } \\
\text { parents) }\end{array}$ & $\sim 29 \%(n=32)$ & $\sim 21 \%(n=32)$ \\
\hline $\begin{array}{l}\text { Birth origin } \\
\text { (captive-born vs. } \\
\text { wild-caught) }^{\mathrm{b}}\end{array}$ & $57 \%(40 / 70)$ & $16 \%(5 / 32)$ \\
\hline $\begin{array}{l}\text { Age at capture } \\
\text { (juvenile vs. adult) }^{\mathrm{b}}\end{array}$ & $55 \%(57 / 103)$ & $19 \%(19 / 101)$ \\
\hline \multirow[t]{2}{*}{$\begin{array}{l}\text { Enrichment (standard } \\
\text { vs. enriched })^{c}\end{array}$} & $58 \%(21 / 36)$ & $\begin{array}{l}13 \%(3 / 24) \\
\text { (enrichment } \\
\text { present) }\end{array}$ \\
\hline & $58 \%(21 / 36)$ & $\begin{array}{l}29 \%(7 / 24) \text { (after } \\
\text { enrichment } \\
\text { removal) }\end{array}$ \\
\hline
\end{tabular}

a The incidence reported is the proportion of each litter of striped mice which developed SB, averaged across litters from each treatment group. The number of litters used in the analyses is provided in parentheses. All fathers were non-stereotypic, and half of the mothers were stereotypic and half non-stereotypic. For ease of the current comparison, data for litters from stereotypic and non-stereotypic dams have been combined The absence/presence of SB was scored when offspring were between 50 and 60 days old (Jones et al., 2010a,b).

b The number of striped mice showing SB in relation to the total number of animals observed is given in parentheses. Birth origin data: the absence/presence of SB in WC striped mice (all captured as adults) was scored thrice - at 4 weeks, 5 months, and 1 year after capture (scores were stable over time). In CB striped mice, SB was scored twice - at 1 month and 5 months after weaning (scores were similarly stable over time). Age at capture date: SB status was scored 4 weeks after capture, by which time all juvenile-caught striped mice were adults (Jones et al., 2011).

c Data from current paper.

is a useful general rule of thumb, the low levels of SB in WC striped mice correlate with poor adjustment to captive conditions (Jones et al., 2011), and therefore the presence of higher levels of motivational frustration (c.f. S/S) is an $a$ priori prediction for E/S subjects: thus even if this motivational frustration is not expressed through the performance of SB, it may impact negatively on welfare-relevant emotional states. In lab. animals, where rearing conditions in commercial production units might differ from housing in research labs., consideration should certainly be given, inter alia, to the sustainability of introduced enrichments to preempt the welfare problems likely induced by enrichment removal (Latham and Mason, 2010).

Overall, our new EE-based model of birth origin effects in striped mice would thus (1) permit the systematic manipulation of the confounding variables of duration of exposure and developmental age; (2) allow for the investigation of untested hypotheses such as whether environmental complexity confers cumulative benefits, or whether animals simply need to receive a certain threshold "dose"; and (3) enable more accurate investigation of if and when during ontogeny sensitive periods may exist for the prevention and/or development of SB in WC animals. If previously enriched striped mice were, as we predict, found to 
be no more fearful once in standard cages than individuals which had always been barren-housed, then a CB model of birth origin effect would have the additional fortuitous consequence of improving the overall welfare of study subjects. Findings from such studies would help inform how captive environments need to be modified to facilitate the development of WC phenotypes in CB animals-so improving the validity of findings from laboratory studies which model behavioural processes observed in the wild, as well as promoting the success of captive-breeding and reintroduction programmes. Findings should also contribute to the development of housing protocols which provide sustainable welfare benefits to wild as well as domestic captive animals.

\section{Conclusion}

Our data showed that enriched-housing from immediately after weaning substantially reduced the incidence of SB in striped mice, and that these beneficial effects persisted (although to a slightly lesser degree) when animals were moved as adults to standard cages. Whilst additional studies are needed to further extend and validate this model, this suggests that an early enrichment protocol could provide a practicable and potentially more ethical means to investigate the causal mechanisms underpinning birth origin effects whereby $C B$ and WC animals differ in behavioural phenotype, especially in terms of SB.

\section{Acknowledgements}

Funding was provided by the National Research Foundation (NRF; grant number: 2069110) and the University of the Witwatersrand. MJ was partially supported whilst collecting data by an NRF Scarce Skills Scholarship. GM was funded by an NSERC Canada Research Chair. Appreciative thanks to Margaret Quinton and Becky Meagher for assistance with the statistical analysis, to Heath Lancaster for having taking the photographs, to Lis Jones and Anne-Marie Lydall for their helpful proofreading, and to two anonymous referees for their constructive input.

\section{References}

Cooper, J.J., Ödberg, F., Nicol, C.J., 1996. Limitations of the effectiveness of environmental improvement in reducing stereotypic behaviour in bank voles (Clethrionomys glareolus). Appl. Anim. Behav. Sci. 48, 237-248.
Garner, J.P., 2006. Perseveration and stereotypy: systems-level insights from clinical psychology. In: Mason, G., Rushen, J. (Eds.), Stereotypic Animal Behaviour: Fundamentals and Applications to Welfare. , second ed. CAB International, Oxford, pp. 121-152.

Hadley, C., Hadley, B., Ephraim, S., Yang, M., Lewis, M.H., 2006. Spontaneous stereotypy and environmental enrichment in deer mice (Peromyscus maniculatus): reversibility of experience. Appl. Anim. Behav. Sci. 97, 312-322.

Jones, M.A., Mason, G., Pillay, N., 2010a. Early social experience influences the development of stereotypic behaviour in captive-born striped mice Rhabdomys. Appl. Anim. Behav. Sci. 123, 70-75.

Jones, M.A., van Lierop, M., Mason, G., Pillay, N., 2010b. Increased reproductive output in stereotypic captive Rhabdomys females: potential implications for captive breeding. Appl. Anim. Behav. Sci. 123, 63-69.

Jones, M.A., Mason, G., Pillay, N., 2011. Correlates of birth origin effects on the development of stereotypic behaviour in striped mice, Rhabdomys. Anim. Behav. 82, 149-159.

Latham, N., Mason, G., 2010. Frustration and perseveration in stereotypic captive animals: is a taste of enrichment worse than none at all? Behav. Brain. Res. 211, 96-104.

Lewis, M.H., Presti, M.F., Lewis, J.B., Turner, C.A., 2006. The neurobiology of stereotypy I: environmental complexity. In: Mason, G., Rushen, J. (Eds.), Stereotypic Animal Behaviour: Fundamentals and Applications to Welfare. , second ed. CAB International, Oxford, pp. 190-226.

Mason, G.J., 1993. Age and context affect the stereotypies of cage mink. Behaviour 127 (3-4), 191-229.

Mason, G., 2006. Are wild-born animals 'protected' from stereotypies? In: Mason, G., Rushen, J. (Eds.), Stereotypic Animal Behaviour: Fundamentals and Applications to Welfare., second ed. CAB International, Oxford, p. 196.

Mason, G., CLubb, R., Latham, N., Vickery, S., 2007. Why and how should we use environmental enrichment to tackle stereotypic behaviour? Appl Anim. Behav. Sci. 102, 163-188.

Mason, G.J., Latham, N., 2004. Can't stop, won't stop: is stereotypy a reliable animal welfare indicator? Anim. Welf. 13, S57-S69.

Ödberg, F.O., 1987. The influence of cage size and environmental enrichment on the development of stereotypies in bank voles (Clethrionomys glareolus). Behav. Proc. 14, 155-173.

Presti, M., Lewis, M., 2005. Striatal opioid peptide content in an animal model of spontaneous stereotypic behavior. Behav. Brain Res. 157, 363-368.

Tilly, S.C., Dallaire, J., Mason, G.J., 2010. Middle-aged mice with enrichment-resistant stereotypic behaviour show reduced motivation for enrichment. Anim. Behav. 80, 363-373.

Turner, C., Yang, M.C., Lewis, M., 2002. Environmental enrichment: effects of stereotyped behaviour and regional metabolic activity. Brain Res. 938, 15-21.

Turner, C., Lewis, M., King, M., 2003. Environmental enrichment effects on stereotyped behaviour and dendritic morphology. Dev. Psychobiol. 43, 20-27.

Vickery, S., Mason, G., 2004. Stereotypic behaviour in Asiatic Black and Malyan Sun Bears. Zoo Biol. 23, 409-430.

Vinke, C.M., 2004. Cage enrichments and the welfare of farmed mink. Ph.D. Thesis. University of Utrecht, Utrecht, Netherlands.

Würbel, H., 2006. The motivational basis of caged rodents' stereotypies. In: Mason, G., Rushen, J. (Eds.), Stereotypic Animal Behaviour: Fundamentals and Applications to Welfare. , second ed. CAB International, Oxford, pp. 86-120.

Zar, J.H., 1996. Biostatistical Analysis, third ed. Prentice Hall, London. 